\title{
Automated VMAT planning for postoperative adjuvant treatment of advanced gastric cancer
}

\author{
Abdul Wahab M. Sharfo ${ }^{1 *} \mathbb{D}$, Florian Stieler², Oskar Kupfer², Ben J. M. Heijmen ${ }^{1}$, Maarten L. P. Dirkx',
} Sebastiaan Breedveld ${ }^{1}$, Frederik Wenz ${ }^{2}$, Frank Lohr ${ }^{3}$, Judit Boda-Heggemann ${ }^{2}$ and Daniel Buergy ${ }^{2}$

\begin{abstract}
Background: Postoperative/adjuvant radiotherapy of advanced gastric cancer involves a large planning target volume (PTV) with multi-concave shapes which presents a challenge for volumetric modulated arc therapy (VMAT) planning. This study investigates the advantages of automated VMAT planning for this site compared to manual VMAT planning by expert planners.
\end{abstract}

Methods: For 20 gastric cancer patients in the postoperative/adjuvant setting, dual-arc VMAT plans were generated using fully automated multi-criterial treatment planning (autoVMAT), and compared to manually generated VMAT plans (manVMAT). Both automated and manual plans were created to deliver a median dose of 45 Gy to the PTV using identical planning and segmentation parameters. Plans were evaluated by two expert radiation oncologists for clinical acceptability. AutoVMAT and manVMAT plans were also compared based on dose-volume histogram (DVH) and predicted normal tissue complication probability (NTCP) analysis.

Results: Both manVMAT and autoVMAT plans were considered clinically acceptable. Target coverage was similar (manVMAT: $96.6 \pm 1.6 \%$, autoVMAT: $97.4 \pm 1.0 \%, p=0.085$ ). With autoVMAT, median kidney dose was reduced on average by > 25\%; (for left kidney from 11.3 \pm 2.1 Gy to $8.9 \pm 3.5$ Gy $(p=0.002)$; for right kidney from $9.2 \pm 2.2$ Gy to $6.1 \pm 1.3 \mathrm{~Gy}(p<0.001))$. Median dose to the liver was lower as well $(18.8 \pm 2.3 \mathrm{~Gy}$ vs. $17.1 \pm 3.6 \mathrm{~Gy}, p=0.048)$. In addition, Dmax of the spinal cord was significantly reduced (38.3 \pm 3.7 Gy vs. $31.6 \pm 2.6 \mathrm{~Gy}, p<0.001)$. Substantial improvements in dose conformity and integral dose were achieved with autoVMAT plans (4.2\% and $9.1 \%$, respectively; $p<0.001)$. Due to the better OAR sparing in the autoVMAT plans compared to manVMAT plans, the predicted NTCPs for the left and right kidney and the liver-PTV were significantly reduced by $11.3 \%, 12.8 \%, 7 \%$, respectively $(p \leq 0.001)$. Delivery time and total number of monitor units were increased in autoVMAT plans (from $168 \pm 19 \mathrm{~s}$ to $207 \pm$ $26 \mathrm{~s}, p=0.006$ ) and (from $781 \pm 168 \mathrm{MU}$ to $1001 \pm 134 \mathrm{MU}, p=0.003$ ), respectively.

Conclusions: For postoperative/adjuvant radiotherapy of advanced gastric cancer, involving a complex target shape, automated VMAT planning is feasible and can substantially reduce the dose to the kidneys and the liver, without compromising the target dose delivery.

Keywords: VMAT, Automated planning, NTCP, Gastric cancer

\footnotetext{
* Correspondence: a.sharfo@erasmusmc.nl

'Department of Radiation Oncology, Erasmus MC Cancer Institute, Groene

Hilledijk 301, 3075, EA, Rotterdam, The Netherlands

Full list of author information is available at the end of the article
}

(c) The Author(s). 2018 Open Access This article is distributed under the terms of the Creative Commons Attribution 4.0 International License (http://creativecommons.org/licenses/by/4.0/), which permits unrestricted use, distribution, and reproduction in any medium, provided you give appropriate credit to the original author(s) and the source, provide a link to the Creative Commons license, and indicate if changes were made. The Creative Commons Public Domain Dedication waiver (http://creativecommons.org/publicdomain/zero/1.0/) applies to the data made available in this article, unless otherwise stated. 


\section{Background}

In the Intergroup Study 0116 (INT-0116) study, adjuvant chemoradiotherapy (CRT) improved overall survival and progression-free survival in patients with gastric cancer compared to surgery alone $[1,2]$. The benefit of combined modality treatment was later confirmed in Asian studies with D2-resected patients [3-5]. European trials in gastroesophageal junction cancers showed survival benefits compared to surgery with neoadjuvant CRT [6] or perioperative chemotherapy without radiotherapy $[7,8]$. Randomized trials comparing adjuvant CRT to (perioperative) chemotherapy indicated clinical benefits for CRT in subgroups, but failed in showing a consistent benefit in European and Asian trials $[3,5,9,10]$. Therefore, the role of adjuvant CRT in gastric carcinoma compared to other approaches, such as perioperative chemotherapy, is not yet clearly defined [9]. The use of modern radiotherapy approaches might improve the risk-/benefit ratio in favor of radiotherapy in gastric carcinoma. Recent data showed the feasibility of using intensity-modulated radiation therapy (IMRT) or volumetric modulated arc therapy (VMAT) for this purpose [3, 10-12]. Postoperative radiotherapy of advanced gastric cancer involves a large target volume with multi-concave shapes which presents a challenge for VMAT planning with a risk of protocol deviations. In a similar setting, radiotherapy for esophageal carcinoma indicated that the experience of the treatment planner may largely affect plan quality, as shown by large differences in plan quality among planners within one institute [13]. In such a setting, automated treatment planning might have several advantages, including facilitated central review in clinical trials as well as improvement of less experienced treatment planner's performance.

Over the years, in-house developed as well as commercial algorithms have attempted to automate the trial-anderror process in order to create optimal plans, reduce user variability and improve the quality and efficiency of the resulting plans. Knowledge-based planning uses a model library of previously generated plans to predict new treatment plan parameters $[14,15]$, while multi-criterial optimization generates a set of Pareto-optimal plans $[16,17]$. Erasmus-iCycle is an optimizer for fully automated multi-criterial beam profile optimization and beam angle selection for coplanar and non-coplanar IMRT, developed at the Erasmus MC Cancer Institute [18-22]. In combination with the Monaco treatment planning system (TPS), Erasmus-iCycle is currently used in clinical practice for IMRT and VMAT plan generation for prostate, head and neck, cervical and lung cancer patients [19-22]. Several studies on postoperative gastric cancer patients have dosimetrically evaluated different radiotherapy techniques [23-26]. To our knowledge, no study has been published considering the feasibility and advantages of automated treatment planning for this treatment site. In this study, we investigated to what extent automated treatment planning using Erasmus-iCycle results in improved VMAT plan quality for advanced gastric cancer patients compared to plan generation by an expert planner.

\section{Methods \\ Patients}

A total of 20 patients with advanced gastric cancer who received radiotherapy treatment were included in the study. Clinical details are shown in Additional file 1: Table S1. Written informed consent was obtained from all patients for anonymized usage of treatment planning data. Our study protocol for retrospective evaluation of automated planning using Erasmus-iCycle was approved by the ethics committee of Heidelberg University, Medical Faculty Mannheim (2016-806R-MA).

\section{Treatment plan generation}

All manually and automatically generated dual-arc VMAT plans (manVMAT and autoVMAT, respectively) were prescribed to deliver a median dose of 45 Gy to the target in 25 fractions. The primary planning objective was achieving adequate PTV coverage while maximally sparing the organs at risk. All plans were generated for delivery at a VersaHD linear accelerator (Elekta AB, Stockholm, Sweden), equipped with an Agility multi-leaf collimator and a photon beam energy of $10 \mathrm{MV}$.

All manVMAT plans were generated by expert treatment planners with the Monaco TPS, version 5.11 (Elekta AB, Stockholm, Sweden) using template-based optimization cost-functions. The employed optimization template is set according to the current clinical guidelines used at the Medical University of Mannheim. For each patient, the optimization cost-functions' parameters were iteratively tweaked to improve the dose distribution and achieve optimal target coverage while respecting OAR constraints. As explained below, also the final autoVMAT plans were generated with Monaco, for which we used the same software version. All Monte Carlo dose calculations in Monaco were performed using a $1 \%$ dose variance, and a dose grid resolution of $3 \mathrm{~mm}$. A maximum of 140 control points per arc was allowed. Also the other segmentation settings were kept identical for manual and automated plan generation.

\section{Automated multi-criterial VMAT plan generation with Erasmus-iCycle/Monaco}

The in-house developed Erasmus-iCycle/Monaco platform for fully automated multi-criteria plan generation [18-22] was configured to generate clinically deliverable VMAT plans for gastric cancer. For each of the study patients, Erasmus-iCycle was used to first automatically generate a Pareto-optimal plan with clinically favorable 
trade-offs between treatment objectives using a "wishlist" developed for gastric cancer (as detailed below in Table 1). Based on this Erasmus-iCycle plan, a patientspecific Monaco template was then created fully automatically, to be used in the Monaco TPS for automated generation of a deliverable dual-arc VMAT plan that mimicked the initial Erasmus-iCycle plan. The applied wish-list contains constraints to be strictly fulfilled, and clinical plan objectives with ascribed priorities to be met as closely as possible or superseded (see Table 1 for details). Three constraints were used to control the maximum dose in the PTV and the patient (i.e., the outline of the patient's external surface including the PTV, delineated OARs and the unspecified tissues), as well as the dose conformity outside the PTV. In order to achieve a homogeneous and adequate PTV dose coverage, the highest priority objective was given to the PTV using the Logarithmic Tumor Control Probability function (LTCP) [27], followed by a shell around the PTV to ensure a steep gradient outside the PTV (priority 2). In line with the clinical practice, minimizing the mean dose in the kidneys was the organ-at-risk (OAR) objective

Table 1 Applied wish-list for automatic VMAT plan generation for gastric cancer patients

\begin{tabular}{|c|c|c|c|c|}
\hline \multicolumn{5}{|l|}{ Constraints } \\
\hline \multicolumn{2}{|l|}{ Volume } & Type & \multicolumn{2}{|l|}{ Limit } \\
\hline \multicolumn{2}{|l|}{ PTV } & Maximum & \multicolumn{2}{|l|}{$105 \%$ of $D^{p}$} \\
\hline \multicolumn{2}{|c|}{ PTV Shell 39 mm } & Maximum & \multicolumn{2}{|l|}{$50 \%$ of $D^{p}$} \\
\hline \multicolumn{2}{|c|}{ Patient } & Maximum & \multicolumn{2}{|l|}{$105 \%$ of $D^{D}$} \\
\hline \multicolumn{5}{|l|}{ Objectives } \\
\hline Priority & Volume & Type & Goal & Parameters \\
\hline 1 & PTV & $\downarrow$ LTCP & 0.4 & $\begin{array}{l}D^{p}=45 \mathrm{~Gy}, \\
a=4\end{array}$ \\
\hline 2 & $\begin{array}{l}\text { PTV Shell } 3 \\
\mathrm{~mm}\end{array}$ & $\downarrow$ Maximum & $90 \%$ of $D^{p}$ & \\
\hline 3 & $\begin{array}{l}\text { Left and Right } \\
\text { Kidney }\end{array}$ & $\downarrow$ Mean & 8 Gy & \\
\hline 4 & Liver & $\downarrow$ Mean & $15 \mathrm{~Gy}$ & \\
\hline 5 & Heart & $\downarrow$ EUD & 15 Gy & $k=6$ \\
\hline 6 & Spinal Cord & $\downarrow$ EUD & 25 Gy & $k=12$ \\
\hline \multirow[t]{2}{*}{7} & $\begin{array}{l}\text { PTV Shell } 18 \\
\text { mm }\end{array}$ & $\downarrow$ Maximum & $40 \%$ of $D^{P}$ & \\
\hline & Skin Ring & $\downarrow$ Maximum & $25 \%$ of $D^{p}$ & $21 \mathrm{~mm}$ \\
\hline \multirow[t]{2}{*}{8} & $\begin{array}{l}\text { Left and Right } \\
\text { Kidney }\end{array}$ & $\begin{array}{l}\downarrow \text { Volume- } \\
\text { Dose }\end{array}$ & $25 \%$ & 12 Gy \\
\hline & Liver & $\begin{array}{l}\downarrow \text { Volume- } \\
\text { Dose }\end{array}$ & $30 \%$ & 24 Gy \\
\hline 9 & $\begin{array}{l}\text { Left and Right } \\
\text { Lung }\end{array}$ & $\begin{array}{l}\downarrow \text { Volume- } \\
\text { Dose }\end{array}$ & $50 \%$ & $20 \mathrm{~Gy}$ \\
\hline
\end{tabular}

Abbreviations: $D^{p}$ prescribed dose, LTCP Logarithmic Tumor Control Probability, $a$ cell sensitivity parameter to achieve adequate target coverage, EUD Equivalent Uniform Dose, $k$ volume parameter with highest priority (priority 3 ), followed by the mean dose in the liver (priority 4). Subsequently, equivalent uniform dose (EUD) objectives with volume effect parameters ( $k=6$ and 12) [28], focusing on reduction of the midrange and high dose in the heart and spinal cord were used (priorities 5 and 6), respectively. To control the dose conformity and entrance dose, a shell at $18 \mathrm{~mm}$ from the PTV, as well as a skin ring of $21 \mathrm{~mm}$ wide from the body contour towards the patient's internal were defined (priority 7). Additionally, dose-volume objectives for the kidneys, liver, and the lungs were used with lower priorities.

\section{Plan evaluation and comparison}

All plans were evaluated by expert radiation oncologists (FL, JBH, and DB) for clinical acceptability. For fair dosimetric plan comparisons, all manVMAT and autoVMAT plans were first normalized to obtain equal median PTV dose (i.e., $\mathrm{D}_{50 \%}=45 \mathrm{~Gy}$ ). In accordance with the International Commission on Radiation Units and Measurements Report No. 83, near-minimum and near-maximum doses ( $D_{98 \%}$ and $D_{2 \%}$, respectively) in the PTV were evaluated, from which the homogeneity index $\left(\mathrm{HI}=\left(\mathrm{D}_{2 \%}\right.\right.$ $\left.\left.\mathrm{D}_{98 \%}\right) / \mathrm{D}_{50 \%}\right)$ was computed. Additionally, dose conformity was estimated by calculating the conformity index (CI $\left.=\left(\mathrm{TV}_{\mathrm{RI}}\right)^{2} /\left(\mathrm{TV}^{*} \mathrm{~V}_{\mathrm{RI}}\right)\right)$, i.e., ratio of the target volume covered by the reference isodose level $\left(\mathrm{TV}_{\mathrm{RI}}\right)$ to the target volume (TV) and volume of the reference isodose $\left(\mathrm{V}_{\mathrm{RI}}\right)$. Quantitative analyses of OAR doses included $D_{\text {mean }}, D_{30 \%}$, $\mathrm{D}_{50 \%}, \mathrm{D}_{60 \%}$ in the kidneys and the liver-PTV, mean and maximum doses $\left(D_{\text {mean }}\right.$, and $\left.D_{\max }\right)$ in the spinal cord and the heart, volumes of the kidneys receiving more than $12 \mathrm{~Gy}\left(\mathrm{~V}_{12 \mathrm{~Gy}}\right)$ and $20 \mathrm{~Gy}\left(\mathrm{~V}_{20 \mathrm{~Gy}}\right)$ [29], volumes of the liver-PTV receiving more than 24 Gy $\left(\mathrm{V}_{24 \mathrm{~Gy}}\right)$ and $30 \mathrm{~Gy}$ $\left(\mathrm{V}_{30 \text { Gy }}\right)$, and $\mathrm{D}_{\text {mean }}$ in the lungs and volumes of the lungs receiving more than 5 Gy $\left(\mathrm{V}_{5 \mathrm{~Gy}}\right)$ and $20 \mathrm{~Gy}\left(\mathrm{~V}_{\text {20Gy }}\right)$ [30]. In addition, integral patient doses were evaluated by assessing patient $D_{\text {mean }}$ and volumes receiving $V_{5 G y}$, $\mathrm{V}_{11.25 \mathrm{~Gy}}$ and $\mathrm{V}_{22.5 \mathrm{~Gy}}$. Estimated treatment delivery time and total number of monitor units (MUs) for each plan were also quantified.

From the DVHs, normal tissue complication probabilities (NTCPs) for the kidneys and the liver were estimated using the Lyman-Burman-Kutcher NTCP model for late effects, considering tolerance dose $\mathrm{TD}_{50 / 5}=12 \mathrm{~Gy}$, $n=0.70, m=0.26$ for the kidneys [29], and $\mathrm{TD}_{50 / 5}=30 \mathrm{~Gy}$, $n=0.32, m=0.15$ for liver failure [31], where $\mathrm{TD}_{50 / 5}$ refers to the dose to the whole organ which lead to complication in $50 \%$ of the population at 5 years, $m$ relates to the steepness of the dose-response curve, and $n$ represents the volume effect in the LKB model. For this purpose, all plans were first normalized to 1.5 Gy per fraction using $\alpha / \beta=2.5$ Gy. 
Differences in dosimetric parameters between manVMAT and autoVMAT plans were analyzed using SPSS software v.21 (SPSS, Inc., Chicago, USA) and presented as the mean \pm 1 standard deviation. Paired two-sided Wilcoxon Signed-rank tests were performed to assess statistical significance of observed differences, considering $p<0.05$ statistically significant.

\section{Results}

\section{Target volume dosimetric evaluations}

All automatically and manually generated VMAT plans were clinically acceptable and achieved adequate target coverage. Clinical acceptability of the plans was evaluated by radiation oncologists with experience in gastric cancer treatment (JBH, FL and DB). Differences between the autoVMAT and manVMAT plans in $\mathrm{V}_{95 \%}$ or $\mathrm{D}_{98 \%}$ were not statistically significant (Table 2). However, autoVMAT plans exhibited a significantly lower near-maximum dose in the PTV, resulting in a better target dose homogeneity $(0.09 \pm 0.01$ vs. $0.10 \pm 0.02(p=0.003))$. For autoVMAT, the dose conformity was significantly improved as well $(0.91 \pm 0.02$ vs. $0.88 \pm 0.03(p<0.001))$. As an example, Fig. 1a shows the resulting dose distributions from the autoVMAT and manVMAT plans for patient 8 . As is evident from this figure and the corresponding dosevolume histograms in Fig. 1b, AutoVMAT resulted in favorable dose conformity and better OAR sparing.

\section{Organs at risk dosimetric evaluations}

Figure 2 shows the observed absolute differences in dosimetric parameters for each of the study patients. Overall, autoVMAT plans had more favorable dose distributions, resulting in reduced dose delivery to the kidneys, liver, spinal cord, heart and lungs, without deteriorating the PTV dose coverage (Table 2 and Fig. 2). Median doses $\left(\mathrm{D}_{50 \%}\right)$ to the left kidney, right kidney, and liver-PTV were significantly reduced by $28 \%$ (from $11.3 \pm 2.1$ Gy to $8.9 \pm 3.5 \mathrm{~Gy}, p=0.002$ ), $39 \%$ (from $9.2 \pm 2.2$ Gy to $6.1 \pm$ $1.3 \mathrm{~Gy}, p<0.001$ ), and $11 \%$ (from $18.8 \pm 2.3$ Gy to 17.1 \pm 3.6 Gy, $p=0.048$ ), respectively. The $\mathrm{V}_{20 \mathrm{~Gy}}$ for the left and right kidney with autoVMAT were lower than those for the manVMAT plans (Table 2). In addition, the $\mathrm{V}_{30 \mathrm{~Gy}}$ for the liver-PTV was significantly decreased with autoVMAT plans by $36 \%$ (from $17.7 \pm 6.2 \%$ to $12.9 \pm 6.0 \%$, $p=0.001$ ). The maximum dose in the spinal cord was on average reduced by $6.7 \pm 3.2$ Gy $(p<0.001)$ with autoVMAT. Furthermore, the integral dose in the patient and the dose conformity were also significantly better with autoVMAT (Table 2).

\section{NTCPs evaluations}

The published sets of parameters used for calculations of NTCPs for the kidneys and liver-PTV resulted in overall lower probability of late complications with
Table 2 Comparison of dosimetric parameters between autoVMAT and manVMAT plans. Population mean values for the 20 study patients and corresponding standard deviations are reported

\begin{tabular}{|c|c|c|c|c|}
\hline \multirow[t]{2}{*}{ Structure } & \multirow[t]{2}{*}{ Parameter } & \multirow{2}{*}{$\begin{array}{l}\text { autoVMAT } \\
\text { Mean } \pm \text { SD }\end{array}$} & \multicolumn{2}{|c|}{ manVMAT - autoVMAT } \\
\hline & & & Mean diff. \pm SD & $p$-value \\
\hline \multirow[t]{5}{*}{ PTV } & $V_{95 \%}(\%)$ & $97.4 \pm 1.0$ & $-0.8 \pm 1.7$ & 0.085 \\
\hline & $\mathrm{D}_{98 \%}(\mathrm{~Gy})$ & $42.5 \pm 0.5$ & $-0.2 \pm 0.7$ & 0.14 \\
\hline & $D_{2 \%}(G y)$ & $46.3 \pm 0.1$ & $0.5 \pm 0.2$ & $<0.001$ \\
\hline & $\mathrm{HI}$ & $0.09 \pm 0.01$ & $0.02 \pm 0.02$ & 0.003 \\
\hline & $\mathrm{Cl}$ & $0.91 \pm 0.02$ & $-0.04 \pm 0.03$ & $<0.001$ \\
\hline \multirow[t]{7}{*}{ Left Kidney } & $D_{\text {mean }}(G y)$ & $12.7 \pm 4.3$ & $1.8 \pm 1.6$ & 0.001 \\
\hline & $\mathrm{D}_{30 \%}(\mathrm{~Gy})$ & $14.9 \pm 8.1$ & $2.1 \pm 3.3$ & 0.013 \\
\hline & $\mathrm{D}_{50 \%}(\mathrm{~Gy})$ & $8.9 \pm 3.5$ & $2.4 \pm 2.2$ & 0.002 \\
\hline & $D_{60 \%}(G y)$ & $7.3 \pm 1.9$ & $2.4 \pm 1.4$ & $<0.001$ \\
\hline & $\mathrm{V}_{12 \mathrm{~Gy}}(\%)$ & $32.2 \pm 14.0$ & $11.7 \pm 7.7$ & $<0.001$ \\
\hline & $V_{20 G y}(\%)$ & $19.2 \pm 13.2$ & $1.0 \pm 3.9$ & 0.117 \\
\hline & NTCP (\%) & $45.1 \pm 35.8$ & $11.3 \pm 10.9$ & 0.001 \\
\hline \multirow[t]{7}{*}{ Right Kidney } & $\mathrm{D}_{\text {mean }}(\mathrm{Gy})$ & $7.5 \pm 1.7$ & $3.0 \pm 1.2$ & $<0.001$ \\
\hline & $D_{30 \%}(G y)$ & $7.8 \pm 1.7$ & $4.1 \pm 1.6$ & $<0.001$ \\
\hline & $\mathrm{D}_{50 \%}(\mathrm{~Gy})$ & $6.1 \pm 1.3$ & $3.1 \pm 1.3$ & $<0.001$ \\
\hline & $\mathrm{D}_{60 \%}(\mathrm{~Gy})$ & $5.5 \pm 1.2$ & $2.7 \pm 1.1$ & $<0.001$ \\
\hline & $V_{12 G y}(\%)$ & $12.4 \pm 4.8$ & $18.9 \pm 8.4$ & $<0.001$ \\
\hline & $V_{20 G y}(\%)$ & $3.8 \pm 2.8$ & $3.0 \pm 2.8$ & 0.001 \\
\hline & NTCP (\%) & $4.2 \pm 3.8$ & $12.8 \pm 7.2$ & $<0.001$ \\
\hline \multirow[t]{7}{*}{ Liver - PTV } & $D_{\text {mean }}(G y)$ & $18.6 \pm 3.3$ & $1.8 \pm 2.8$ & 0.02 \\
\hline & $\mathrm{D}_{30 \%}(\mathrm{~Gy})$ & $21.9 \pm 4.1$ & $2.8 \pm 3.2$ & 0.003 \\
\hline & $\mathrm{D}_{50 \%}(\mathrm{~Gy})$ & $17.1 \pm 3.6$ & $1.7 \pm 3.3$ & 0.048 \\
\hline & $D_{60 \%}(G y)$ & $15.1 \pm 3.4$ & $1.2 \pm 3.3$ & 0.14 \\
\hline & $V_{24 G y}(\%)$ & $25.4 \pm 11.6$ & $7.1 \pm 8.8$ & 0.004 \\
\hline & $V_{30 G y}(\%)$ & $12.9 \pm 6.0$ & $4.7 \pm 4.6$ & 0.001 \\
\hline & NTCP (\%) & $31.1 \pm 23.2$ & $7.0 \pm 7.0$ & 0.001 \\
\hline \multirow[t]{2}{*}{ Heart } & $D_{\text {mean }}(G y)$ & $12.7 \pm 4.7$ & $0.7 \pm 1.8$ & 0.117 \\
\hline & $D_{\max }(G y)$ & $46.8 \pm 0.6$ & $0.8 \pm 1.2$ & 0.009 \\
\hline \multirow[t]{2}{*}{ Spinal cord } & $D_{\text {mean }}(G y)$ & $13.9 \pm 2.6$ & $2.9 \pm 1.5$ & $<0.001$ \\
\hline & $D_{\max }(G y)$ & $31.6 \pm 2.6$ & $6.7 \pm 3.2$ & $<0.001$ \\
\hline \multirow[t]{3}{*}{ Left Lung } & $D_{\text {mean }}(G y)$ & $7.3 \pm 3.2$ & $0.6 \pm 0.3$ & $<0.001$ \\
\hline & $V_{5 G y}(\%)$ & $32.7 \pm 15.6$ & $1.4 \pm 1.0$ & $<0.001$ \\
\hline & $V_{20 G y}(\%)$ & $14.0 \pm 7.2$ & $2.2 \pm 1.8$ & $<0.001$ \\
\hline \multirow[t]{3}{*}{ Right Lung } & $D_{\text {mean }}(G y)$ & $5.4 \pm 3.3$ & $0.3 \pm 0.5$ & 0.013 \\
\hline & $V_{5 G y}(\%)$ & $31.2 \pm 16.8$ & $1.1 \pm 2.1$ & 0.01 \\
\hline & $V_{20 G y}(\%)$ & $7.0 \pm 7.6$ & $0.8 \pm 1.8$ & 0.078 \\
\hline \multirow[t]{4}{*}{ Patient } & $D_{\text {mean }}(G y)$ & $9.4 \pm 1.9$ & $0.5 \pm 0.3$ & $<0.001$ \\
\hline & $V_{5 G y}(\%)$ & $40.2 \pm 8.9$ & $1.6 \pm 0.8$ & $<0.001$ \\
\hline & $V_{11.25 G y}(\%)$ & $30.0 \pm 6.6$ & $1.8 \pm 1.7$ & 0.001 \\
\hline & $V_{22.5 G y}(\%)$ & $16.2 \pm 3.3$ & $1.5 \pm 1.1$ & $<0.001$ \\
\hline
\end{tabular}

Abbreviations: $\mathrm{HI}$ homogeneity index, $\mathrm{Cl}$ conformity index, NTCP normal tissue complication probability 


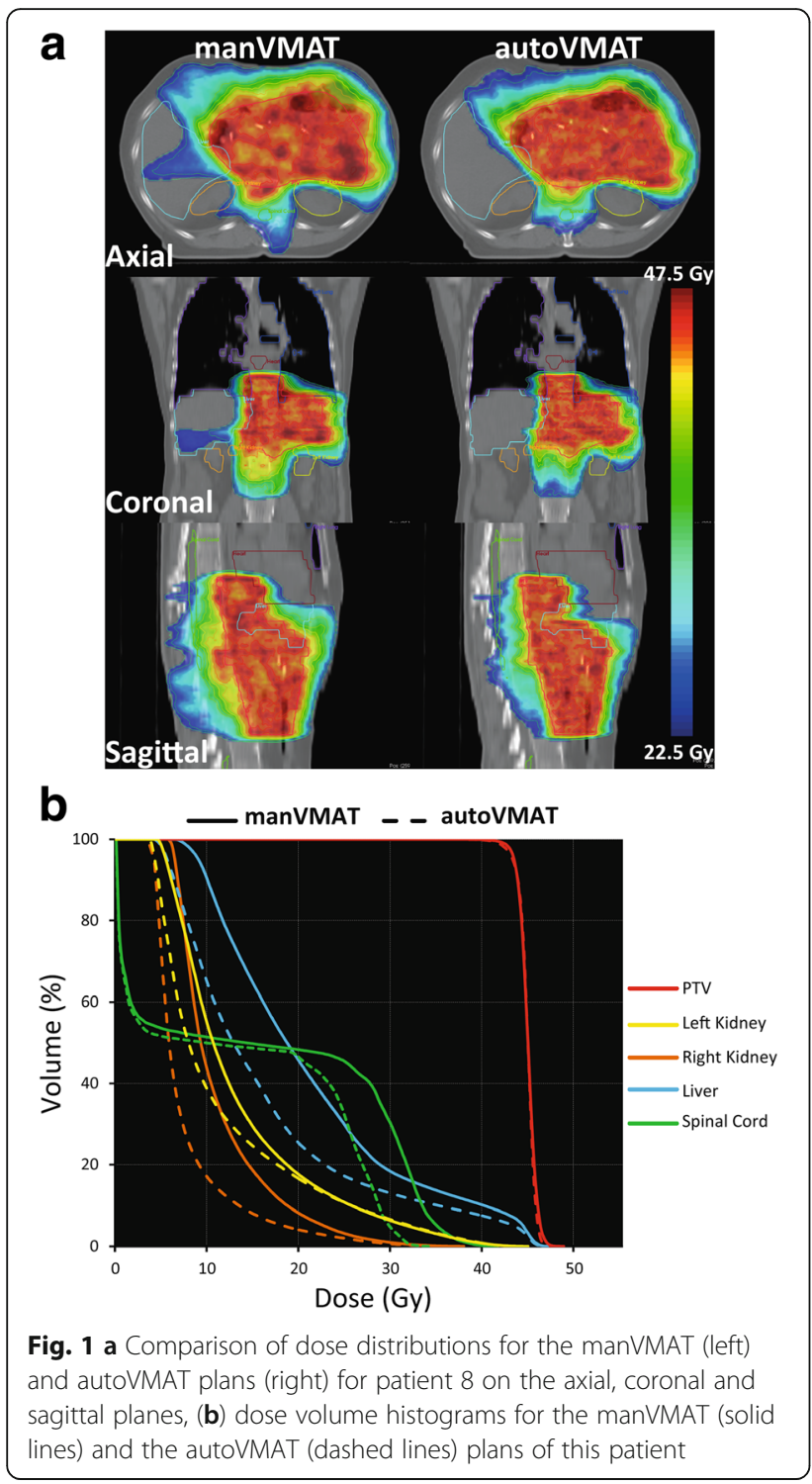

autoVMAT compared to manVMAT plans. In $16 / 20$ patients, the predicted NTCPs were lowest with autoVMAT for the left kidney, whereas all 20 patients had lower predicted NTCPs with autoVMAT for the right kidney (Fig. 3). For $17 / 20$ patients, the NTCP predictions for liver-PTV were lower for the autoVMAT plans. The average NTCP values resulting from autoVMAT, given in Table 2 , were significantly reduced by $11.3 \%$, $12.8 \%$ and $7 \%$ for the left and right kidney and the liver-PTV, respectively.

\section{Planning and treatment delivery times}

All manVMAT plans in this study were generated using template-based manual planning. Hands-on tweaking time of the optimization cost functions was on average $30 \mathrm{~min}$ (range 5-60 min). The optimization and dose calculation of autoVMAT plans in Erasmus-iCycle/Monaco was fully automated and therefore did not require any hands-on time.

Compared to manVMAT plans a significantly more MUs were required for delivery of the autoVMAT plans (1001 $\pm 134 \mathrm{MU}$ vs. $781 \pm 168 \mathrm{MU}, p=0.003$ ), resulting in a prolonged (estimated) treatment delivery time (207 \pm 26 s vs. $168 \pm 19$ s, $p=0.006$ ).

\section{Discussion}

Gastric cancer is the third leading cause of cancer death in both sexes with yearly 723,000 deaths worldwide [32]. Protocol deviations can reduce clinical efficacy of radiotherapy in complex treatment geometries, like gastric carcinoma. In the centralized review of the INT-0116 study, minor or major protocol deviations were observed in $35 \%$ of patients [2]. Even after correction of errors, major protocol deviations were still found in $6.5 \%$ of the plans that were actually irradiated $[1,2]$. The clinical impact of protocol deviations in INT-0116 has not been reported. However, in other complex geometries it was shown that protocol violations during radiation therapy were correlated with reduced overall survival [33]. Modern radiotherapy approaches such as IMRT and VMAT have several dosimetric advantages but they are associated with increased complexity [9, 34-36]. Very high protocol deviations are therefore possible; for example, RTOG 0529 showed $81 \%$ protocol deviations at first plan review [37]. Albeit in small numbers, RTOG 0022 showed local failure rates of $50 \%(2 / 4)$ vs. $6 \%(3 / 49)$ in oropharyngeal cancer patients treated with and without protocol deviations, respectively [38]. Protocol deviations may, amongst other things, be caused by inaccurate delineation or sub-optimal treatment planning. For gastric cancer, the percentage of these deviations is unknown. In a recent report on RTOG 0933 by Gondi et al. [39], unacceptable radiotherapy protocol deviations were observed in $25 \%$ of cases at a rapid review process: $11 / 21$ cases had contouring deviations, $5 / 21$ cases had unacceptable planning deviations and $5 / 21$ had unacceptable deviations of contouring and planning. Also in a recent study of Habraken et al. [40] on hepatocellular carcinoma patients, it was demonstrated that pretreatment plan review was important to reduce unacceptable protocol deviations. Additionally, they demonstrated that automated treatment planning could be used to identify sub-optimal treatment plans. This might be especially relevant in trials in which low numbers of patients are eligible or small numbers of patients are treated in a participating hospital.

Over the past years, several studies have investigated the advantages of automated treatment planning compared to manual planning using in-house developed or commercial algorithms [18-22, 41-43]. Treatment planning with Erasmus-iCycle/Monaco is fully automated, 


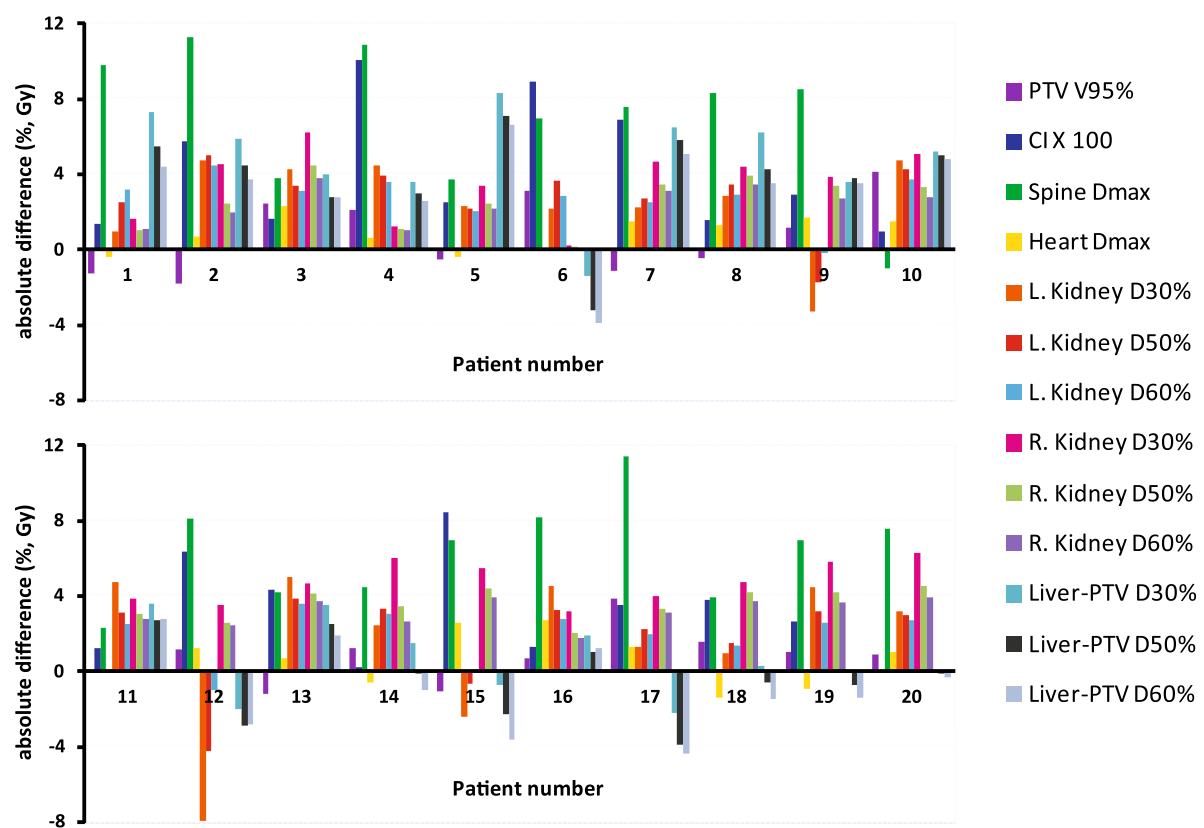

Fig. 2 Differences in dosimetric plan parameters between autoVMAT and manVMAT plans for each of the 20 study patients. Positive values are in favor of the autoVMAT plans

and it has been successfully validated for clinical use in several treatment sites including prostate [19], headand-neck [20], cervix [21], and lung [22]. Knowledgebased planning using a model library of previously generated plans to predict treatment plan parameters for a new patient was configured and tested in pelvic anatomy [41], lung [42], and esophageal cancer [43].

Numerous dosimetric studied have evaluated different radiotherapy techniques for gastric cancer by comparing IMRT, VMAT, helical tomotherapy, 3DCRT and proton therapy [23-26]. Until now, no study has been published showing the possibility/advantages of automated treatment planning for postoperative gastric cancer patients. In this study Erasmus-iCycle was used to automatically generate VMAT plans for gastric cancer patients. For this site, manual treatment optimization is a large challenge, even for an expert planner, due to the multiconcave shape, the extent of the target volume, and the close proximity to many radiosensitive organs (i.e., kidneys, liver, heart, and spinal cord). Another difficulty in postoperative radiotherapy in gastric cancer patients is that radiation tolerance doses of OARs are relatively low. Compared to the manVMAT plans, generated by an expert planner, plan quality was significantly better for the autoVMAT plans, only requiring slightly longer treatment delivery times. Specifically, dose conformity and sparing of organs at risk were improved, while the clinical importance of the observed longer treatment delivery time and the increased MUs is considered to be low. As a result of higher modulation, the number

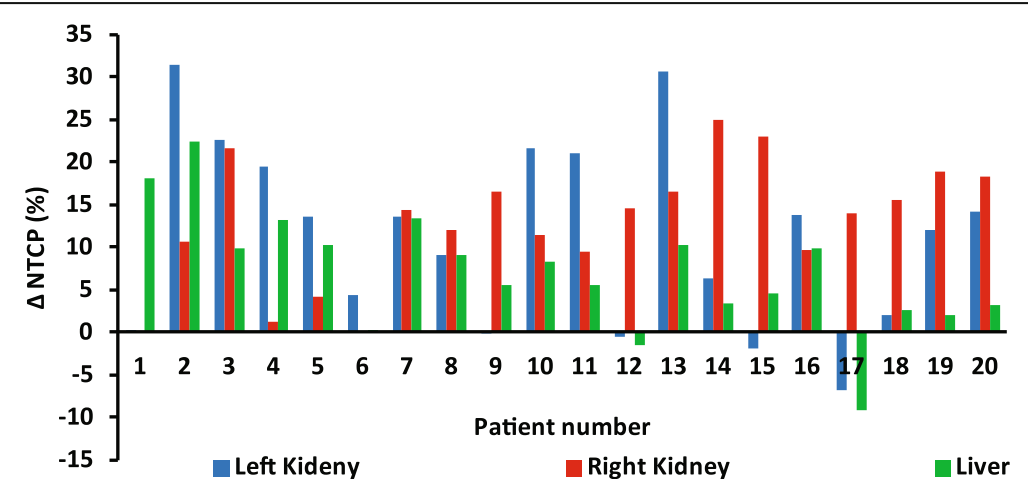

Fig. 3 Differences in the predicted normal tissue complication probabilities (NTCP) between autoVMAT and manVMAT plans for the 20 study patients. Positive values are in favor of the autoVMAT plans 
of MUs was significantly higher in the autoVMAT than in manVMAT plans, this could lead to challenges in radiation delivery. The integral dose was significantly reduced in autoVMAT plans, this may be beneficial specially for young patients to avoid secondary tumors, and most notably showed significantly reduced NTCP for liver and both kidneys, although it is not yet clear to what extent the observed dosimetric advantages of autoVMAT vs. an experienced planner will really translate into a clinical benefit. Automated treatment planning can improve the efficiency of the treatment planning process and reduce its user dependency, this in turns might lead to more consistent and uniform outcomes in treatment planning studies and clinical trials. Apart from the improved plan quality and reduced predicted complications, automated planning also eliminated the planning hands-on time required for planning.

\section{Conclusion}

Automated treatment planning is of great value for complex treatment sites like for gastric cancer. Compared to manual planning by an expert planner, plan quality could be largely improved, while drastically reducing treatment planning workload.

\section{Additional file}

Additional file 1: Table S1. Clinical details of patients with gastric carcinoma. (DOCX $15 \mathrm{~kb}$ )

\begin{abstract}
Abbreviations
autoVMAT: Fully automated volumetric modulated arc therapy (plans); Cl: Conformity index; CRT: Chemoradiotherapy; DVH: Dose-volume histogram; EUD: Equivalent uniform dose; HI: Homogeneity index; IMRT: Intensity-modulated radiation therapy; INT-0116: Intergroup Study 0116; LTCP: Logarithmic tumor control probability; manVMAT: Manually generated volumetric modulated arc therapy (plans); MU: Monitor units; NTCP: Normal tissue complication probability; OAR: Organ at risk; PTV: Planning target volume; RTOG: Radiation Therapy Oncology Group; TPS: Treatment planning system; TV: Target volume; VMAT: Volumetric modulated arc therapy
\end{abstract}

\section{Funding}

This work was in part funded by a research grant of Elekta AB, Stockholm, Sweden

\section{Availability of data and materials}

The dataset generated and analyzed during the current study are available from the corresponding author on reasonable request.

\section{Authors' contributions}

$J B H, D B$, and AWMS conceived the study; BJMH, MLPD, and SB provided supervision and assistance with iCycle procedures; $\mathrm{OK}, \mathrm{JBH}$, and DB re-contoured OAR and target volumes, if appropriate; FS re-planned clinically irradiated plans with modern planning systems; FW and FL provided assistance with study design and revisions of the manuscript; All authors read and approved the final manuscript.

\section{Ethics approval and consent to participate}

The study was approved by the ethics committee of Heidelberg University, Medical Faculty Mannheim (2016-806R-MA). Written informed consent for scientific usage of clinical data was obtained from all patients.

\section{Consent for publication}

Consent for scientific usage of clinical data was obtained from all patients included in the study.

\section{Competing interests}

Erasmus MC Cancer Institute has research collaborations with Accuray Inc., Sunnyvale, USA.

AWMS reports grants from Elekta AB, Stockholm, Sweden, during the conduct of the study; FS reports personal fees from Elekta AB, Sweden, outside the submitted work; OK has nothing to disclose: BJMH reports grants from Elekta $A B$, Stockholm, Sweden, during the conduct of the study; grants from Accuray Inc., Sunnyvale, USA, outside the submitted work; MLPD reports grants from Elekta AB, Stockholm, Sweden, during the conduct of the study; SB reports grants from Elekta $A B$, Stockholm, Sweden, during the conduct of the study; grants from Accuray Inc., Sunnyvale, USA, outside the submitted work; FW reports research grants and travel support from Elekta, during the conduct of the study; FL has nothing to disclose; JBH reports teaching honoraria from Elekta AB, Stockholm, Sweden, during the conduct of the study; DB reports personal fees from Siemens AG, personal fees from NB Capital ApS, outside the submitted work.

\section{Publisher's Note}

Springer Nature remains neutral with regard to jurisdictional claims in published maps and institutional affiliations.

\section{Author details}

${ }^{1}$ Department of Radiation Oncology, Erasmus MC Cancer Institute, Groene Hilledijk 301, 3075, EA, Rotterdam, The Netherlands. ${ }^{2}$ Universitätsmedizin Mannheim, Medical Faculty Mannheim, Heidelberg University, Mannheim, Germany. ${ }^{3}$ Unita Operativa di Radioterapia, Dipartimento di Oncologia, Az. Ospedaliero-Universitaria di Modena, Modena, Italy.

Received: 27 February 2018 Accepted: 16 April 2018

Published online: 23 April 2018

\section{References}

1. Macdonald JS, Smalley SR, Benedetti J, et al. Chemoradiotherapy after surgery compared with surgery alone for adenocarcinoma of the stomach or gastroesophageal junction. N Engl J Med. 2001;345:725-30.

2. Smalley SR, Benedetti JK, Haller DG, et al. Updated analysis of SWOG-directed intergroup study 0116: a phase III trial of adjuvant radiochemotherapy versus observation after curative gastric cancer resection. J Clin Oncol. 2012;30:2327-33.

3. Zhu WG, Xua DF, Pu J, et al. A randomized, controlled, multicenter study comparing intensity-modulated radiotherapy plus concurrent chemotherapy with chemotherapy alone in gastric cancer patients with D2 resection. Radiother Oncol. 2012;104:361-6.

4. Lee J, Lim DH, Kim S, et al. Phase III trial comparing capecitabine plus cisplatin versus capecitabine plus cisplatin with concurrent capecitabine radiotherapy in completely resected gastric cancer with D2 lymph node dissection: the ARTIST trial. J Clin Oncol. 2012;30:268-73.

5. Park SH, Sohn TS, Lee J, et al. Phase III trial to compare adjuvant chemotherapy with Capecitabine and cisplatin versus concurrent chemoradiotherapy in gastric Cancer: final report of the adjuvant chemoradiotherapy in stomach tumors trial, including survival and subset analyses. J Clin Oncol. 2015;33:3130-6.

6. van Hagen P, Hulshof MC, van Lanschot JJ, et al. Preoperative chemoradiotherapy for esophageal or junctional cancer. N Engl J Med. 2012;366:2074-84

7. Ychou M, Boige V, Pignon JP, et al. Perioperative chemotherapy compared with surgery alone for resectable gastroesophageal adenocarcinoma: an FNCLCC and FFCD multicenter phase III trial. J Clin Oncol. 2011;29:1715-21.

8. Cunningham D, Allum WH, Stenning SP, et al. Perioperative chemotherapy versus surgery alone for resectable gastroesophageal cancer. N Engl J Med. 2006:355:11-20.

9. Buergy $D$, Lohr F, Baack T, et al. Radiotherapy for tumors of the stomach and gastroesophageal junction-a review of its role in multimodal therapy. Radiat Oncol. 2012;7:192. 
10. Verheij $\mathrm{M}$, Jansen $\mathrm{E}$, Cats $\mathrm{A}$, et al. A multicenter randomized phase III trial of neo-adjuvant chemotherapy followed by surgery and chemotherapy or by surgery and chemoradiotherapy in resectable gastric cancer: first results from the CRITICS study. J Clin Oncol. 2016;34(15_suppl):4000. https://doi. org/10.1200/JCO.2016.34.15_suppl.4000.

11. Boda-Heggemann J, Weiss C, Schneider V, et al. Adjuvant IMRT/XELOX radiochemotherapy improves long-term overall- and disease-free survival in advanced gastric cancer. Strahlenther Onkol. 2013;189:417-23.

12. Dikken JL, van Sandick JW, Maurits Swellengrebel HA, et al. Neo-adjuvant chemotherapy followed by surgery and chemotherapy or by surgery and chemoradiotherapy for patients with resectable gastric cancer (CRITICS). BMC Cancer. 2011;11:329.

13. Berry SL, Boczkowski A, Ma R, Mechalakos J, Hunt M. Interobserver variability in radiation therapy plan output: results of a single-institution study. Pract Radiat Oncol. 2016;6:442-9.

14. Appenzoller LM, Michalski JM, Thorstad WL, Mutic S, Moore KL. Predicting dose-volume histograms for organs-at-risk in IMRT planning. Med Phys. 2012;39:7446-61.

15. Yuan L, Ge Y, Lee WR, Yin FF, Kirkpatrick JP, Wu QJ. Quantitative analysis of the factors which affect the interpatient organ-at-risk dose sparing variation in IMRT plans. Med Phys. 2012;39:6868-78.

16. Monz M, Kufer KH, Bortfeld TR, Thieke C. Pareto navigation: algorithmic foundation of interactive multi-criteria IMRT planning. Phys Med Biol. 2008;53:985-98

17. Breedveld S, Storchi PR, Keijzer M, Heemink AW, Heijmen BJM. A novel approach to multi-criteria inverse planning for IMRT. Phys Med Biol. 2007:52:6339-53.

18. Breedveld S, Storchi PRM, Voet PWJ, Heijmen BJM. Icycle: integrated, multicriterial beam angle, and profile optimization for generation of coplanar and noncoplanar IMRT plans. Med Phys. 2012;39:951-63.

19. PWJ V, MLP D, Breedveld S, Al-Mamgani A, Incrocci L, BJM H. Fully automated volumetric modulated arc therapy plan generation for prostate cancer patients. Int J Radiat Oncol Biol Phys. 2014;88:1175-9.

20. Voet PWJ, Dirkx MLP, Breedveld S, Fransen D, Levendag PC, Heijmen BJM. Toward fully automated multicriterial plan generation: a prospective clinical study. Int J Radiat Oncol Biol Phys. 2013;85:866-72.

21. Sharfo AWM, Breedveld S, Voet PWJ, et al. Validation of fully automated VMAT plan generation for library-based plan-of-the-day cervical cancer radiotherapy. PLoS One. 2016;11:e0169202.

22. Della Gala G, Dirkx ML, Hoekstra N, et al. Fully automated VMAT treatment planning for advanced-stage NSCLC patients. Strahlenther Onkol. 2017;193:402-9.

23. Li Z, Zeng J, Wang Z, Zhu H, Wei Y. Dosimetric comparison of intensity modulated and volumetric arc radiation therapy for gastric cancer. Oncol Lett. 2014;8:1427-34

24. Zhang T, Liang ZW, Han J, Bi JP, Yang ZY, Ma H. Double-arc volumetric modulated therapy improves dose distribution compared to static gantry IMRT and 3D conformal radiotherapy for adjuvant therapy of gastric cancer. Radiat Oncol. 2015:19:114.

25. Onal C, Dolek Y, Akkus YB. Dosimetric comparison of 3-dimensional conformal radiotherapy, volumetric modulated arc therapy, and helical tomotherapy for postoperative gastric cancer patients. Jpn J Radiol. 2018;36:30-9.

26. Mondlane G, Gubanski M, Lind PA, Ureba A, Siegbahn A. Comparison of gastric-cancer radiotherapy performed with volumetric modulated arc therapy or single-field uniform-dose proton therapy. Acta Oncol. 2017; 56:832-8.

27. Alber M, Reemtsen R. Intensity modulated radiotherapy treatment planning by use of a barrier-penalty multiplier method. Opt Methods Softw. 2007;22:391-411.

28. Niemierko A. Reporting and analyzing dose distributions: a concept of equivalent uniform dose. Med Phys. 1997;24:103-10.

29. Dawson LA, Kavanagh BD, Paulino AC, et al. Radiation-associated kidney injury. Int J Radiat Oncol Biol Phys. 2010;76:S108-15.

30. Marks LB, Bentzen SM, Deasy JO, et al. Radiation dose-volume effects in the lung. Int J Radiat Oncol Biol Phys. 2010;76:S70-6.

31. Dawson LA, Normolle D, Balter JM, McGinn CJ, Lawrence TS, Ten Haken RK. Analysis of radiation-induced liver disease using the Lyman NTCP model. Int J Radiat Oncol Biol Phys. 2002;53:810-21.

32. Ferlay J, Soerjomataram I, Dikshit R, et al. Cancer incidence and mortality worldwide: sources, methods and major patterns in GLOBOCAN 2012. Int J Cancer. 2015;136:E359-86.
33. Wong KK, All S, Waxer J, et al. Radiotherapy after high-dose chemotherapy with autologous hematopoietic cell rescue: quality assessment of head start III. Pediatr Blood Cancer. 2017;64:e26529.

34. Wieland P, Dobler B, Mai S, et al. IMRT for postoperative treatment of gastric cancer: covering large target volumes in the upper abdomen: a comparison of a step-and-shoot and an arc therapy approach. Int J Radiat Oncol Biol Phys. 2004;59:1236-44

35. Lohr F, Dobler B, Mai S, et al. Optimization of dose distributions for adjuvant locoregional radiotherapy of gastric cancer by IMRT. Strahlenther Onkol. 2003;179:557-63.

36. Haneder S, Michaely HJ, Schoenberg SO, et al. Assessment of renal function after conformal radiotherapy and intensity-modulated radiotherapy by functional 1H-MRI and 23Na-MRI. Strahlenther Onkol. 2012;188:1146-54.

37. Kachnic LA, Winter K, Myerson RJ, et al. RTOG 0529: a phase 2 evaluation of dose-painted intensity modulated radiation therapy in combination with 5fluorouracil and mitomycin-C for the reduction of acute morbidity in carcinoma of the anal canal. Int J Radiat Oncol Biol Phys. 2013;86:27-33.

38. Eisbruch A, Harris J, Garden AS, et al. Multi-institutional trial of accelerated hypofractionated intensity-modulated radiation therapy for early-stage oropharyngeal cancer (RTOG 00-22). Int J Radiat Oncol Biol Phys. 2010;76:1333-8.

39. Gondi V, Cui Y, Mehta MP, et al. Real-time pretreatment review limits unacceptable deviations on a cooperative group radiation therapy technique trial: quality assurance results of RTOG 0933. Int J Radiat Oncol Biol Phys. 2015;91:564-70.

40. Habraken SM, Sharfo AW, Buijsen J, et al. The TRENDY multi-center randomized trial on hepatocellular carcinoma - trial QA including automated treatment planning and benchmark-case results. Radiother Oncol. 2017;125:507-13

41. Hussein M, South CP, Barry MA, et al. Clinical validation and benchmarking of knowledge-based IMRT and VMAT treatment planning in pelvic anatomy Radiother Oncol. 2016;120:473-9.

42. Fogliata A, Belosi F, Clivio A, et al. On the pre-clinical validation of a commercial model-based optimization engine: application to volumetric modulated arc therapy for patients with lung or prostate cancer. Radiother Oncol. 2014;113:385-91.

43. Fogliata A, Nicolini G, Clivio A, et al. A broad scope knowledge based model for optimization of VMAT in esophageal cancer: validation and assessment of plan quality among different treatment centers. Radiat Oncol. 2015;10:220

\section{Ready to submit your research? Choose BMC and benefit from:}

- fast, convenient online submission

- thorough peer review by experienced researchers in your field

- rapid publication on acceptance

- support for research data, including large and complex data types

- gold Open Access which fosters wider collaboration and increased citations

- maximum visibility for your research: over $100 \mathrm{M}$ website views per year

At BMC, research is always in progress.

Learn more biomedcentral.com/submissions 